\title{
Lamin B1 deficiency promotes malignancy and predicts poor prognosis in gastric cancer
}

\author{
Z. Y. YU ${ }^{1, *}$, X. Y. JIANG ${ }^{1, *}$, R. R. ZHAO ${ }^{2}$, C. J. LUO ${ }^{1}$, Y. X. REN ${ }^{1}$, Z. J. MA ${ }^{3}$, H. L. YE ${ }^{3}$, W. G. SHI ${ }^{3}$, C. WANG ${ }^{1}$, Z. Y. JIAO 1,3,*
}

${ }^{1}$ Department of General Surgery, Lanzhou University Second Hospital, Lanzhou University, Lanzhou, Gansu, China; ${ }^{2}$ Department of Infectious Diseases, The First Hospital of Lanzhou University, Lanzhou University, Lanzhou, Gansu, China; ${ }^{3}$ Cui-ying Experimental Center, Lanzhou University Second Hospital, Lanzhou University, Lanzhou, Gansu, China

${ }^{*}$ Correspondence: jiaozy@lzu.edu.cn

${ }^{*}$ Contributed equally to this work.

Received January 9, 2020 / Accepted June 23, 2020

\begin{abstract}
Gastric cancer (GC) is a kind of global malignancy. However, the expression pattern and clinical relevance of lamin B1 in GC remain to be elucidated. We endeavored to investigate how GC is influenced by lamin B1 and the related mechanisms. The lamin B1 expression in GC tissues from 71 patients was assessed by using immunohistochemistry (IHC). The expression of lamin B1 was connected with the clinical stage, depth of invasion, and poorer overall survival. Colony formation assays and methyl thiazolyl tetrazolium (MTT) were used to assess cell viability. The migration ability of GC cells was determined by cell scratch assay and Transwell invasion assay. Moreover, we used two cell lines of GC to explore the underlying mechanism of lamin B1 in boosting the GC cells proliferation and invasion in vitro by assessing the effects on related signal transduction pathways. Our data demonstrated that the expression level of lamin B1 was downregulated in GC tissues, and low expression level of lamin B1 was significantly correlated with higher clinical stage, depth of invasion, nodal stage, and poor prognosis. Moreover, in vitro experiments demonstrated that lamin B1 knockdown promoted, whereas lamin B1 overexpression inhibited, gastric cancer cell proliferation and migration. We also observed that lamin B1 knockdown could promote the activity of the PI3K/PTEN/Akt and MAPK/ERK pathway with a decrease in the p53/p21WAF1/CIP1 expression, whereas lamin B1 overexpression contributed to the opposite results. In conclusion, our studies indicate that lamin B1 deficiency is crucial in GC progression. Furthermore, the results elucidating the biological mechanisms of lamin B1 may potentially contribute to current GC treatment modalities.
\end{abstract}

Key words: lamin B1, gastric cancer, signal transduction

As a common malignancy in humans, gastric cancer (GC) is still one of the main causes of cancer-related death globally [1]. Its pathogenesis has not been fully elucidated and effective targeted therapeutics are still lacking [2]. For GC treatment, one of the main approaches is chemotherapy. However, because of its nonspecific antitumor effect, the therapeutic efficacy is quite limited and often accompanied by serious toxic side effects. Even with the first-line chemotherapy regimen, the overall response rate is still lower than $50 \%$ for GC patients with radical gastrectomy combined with chemotherapy [3], and the median survival time is only 6.6-18.4 months for unresectable GC patients [4]. Therefore, developing a new therapeutic target to increase the clinical outcomes of GC patients is urgent.

Lamin B1, encoded by LMNB1, is a kind of lamins protein and a key component in forming the nuclear lamina. This protein is fundamental for human health, as it maintains the functional and structural integrity of the nuclear envelope in higher eukaryotic cells. Lamin B1 not only anchors certain genome areas to the nuclear periphery but also functions in chromosome positioning $[5,6]$, affecting DNA repair, replication, and transcription [7]. Current studies have shown that lamin $\mathrm{B} 1$ is involved in biological processes, e.g., proliferation and migration in various tumor cells $[8,9]$. However, there are no functional, clinicopathological, or prognostic studies based on lamin B1 in GC. In this study, we endeavored to elucidate lamin B1's functions in GC cells and elucidate its antitumor role. Our data indicate that lamin B1 can be a promising factor of prognosis in GC patients as well as a potential tumor suppressor gene.

\section{Patients and methods}

Samples. Patients between May 2014 and December 2015 at Lanzhou University Second Hospital provided the paraffin-embedded samples. Sufficiently respecting the 
patients' right to informed consent, we used these samples under the permission of the Medical Ethics Committee of Lanzhou University Second Hospital. There were 71 primary GC patients in this study: 49 males and 22 females (average year, 50; range, 21-75 years), all who received no pretreatment (radiotherapy or chemotherapy) before the surgery. To eliminate the interference, we kept GC tissues $(\mathrm{n}=71)$ and normal tissues $(\mathrm{n}=31)$ at $-80^{\circ} \mathrm{C}$ in liquid nitrogen until this study started. Based on the UICC classification (TNM 2010), every specimen was verified by pathological examination and subjected to TNM staging.

Cell lines and cell culture. Two GC lines (SGC-7901 and MGC-803) were authenticated by short tandem repeat (STR) fingerprinting, and they were purchased from the Cell Bank of Type Culture Collection of the Chinese Academy of Sciences (Shanghai, China). We cultured the cells in Dulbecco's modified Eagle's medium (DMEM) (Gaithersburg, USA) with added $10 \%$ heat-inactivated fetal calf serum (Logan, UT) at $5 \% \mathrm{CO}_{2}$ and $37^{\circ} \mathrm{C}$ in a humidified incubator. Furthermore, the expression of lamin B1 was assessed by western blotting. We used $\beta$-actin as a reference.

siRNA transfection. siRNA was used to inhibit the expression of lamin B1. Lamin B1 siRNA was synthesized by GenePharma (Shanghai, China), and the sequences used are shown as follows: siRNA-1, sense: 5'-GCAGACUUACCAUGCCAAA-3' and antisense: 5'-UUUGGCAUGGUAAGUCUGC-3'; siRNA 2, sense: 5'-GGAGACACAUCAGUCAGUUTT-3' and antisense: 5'-AACUGACUGAUGUGUCUCCTT-3'; and siRNA 3, sense: 5'-GGCGGAAAUAAGGGAUCAA-3' and antisense: 5'-UUGAUCCCUUAUUUCCGCC-3'. After PCR and western blot analyses, we selected the final two target sequences with the best silencing efficiency on lamin B1 expression after the competition as siRNA 1 and siRNA 2, and we selected a corresponding negative control sequence. Assays for evaluating gene silencing efficiency were performed by western blot and qRT-PCR $48 \mathrm{~h}$ after transfection. After $48 \mathrm{~h}$, we harvested the cells for analysis.

Lamin B1 overexpression. To express lamin B1 in gastric cancer cells, we constructed a plasmid of pcDNA3.1-lamin B1 and transfected the plasmid into MGC-803 and SGC-7901 by Lipofectamine 2000 . After $48 \mathrm{~h}$, we harvested the cells to detect expression efficiency by western blot assay and the ability of proliferation and migration.

Quantitative real-time fluorescent PCR (qRT-PCR). A PrimeScript RT reagent Kit (TAKARA, USA) was used to synthesized complementary DNA from total RNA. Also, we performed a qRT-PCR with SYBR Premix ExTaq (TAKARA, USA). To normalize the relative expression of lamin B1, we took the endogenous $18 \mathrm{~S}$ rRNA expression as the control and calculated the $-\Delta C T$ value. The qRT-PCR conditions are shown as follows: $60^{\circ} \mathrm{C}$ for $34 \mathrm{~s}, 60^{\circ} \mathrm{C}$ for $60 \mathrm{~s}, 40$ cycles at $95^{\circ} \mathrm{C}$ for $5 \mathrm{~s}, 95^{\circ} \mathrm{C}$ for $15 \mathrm{~s}$, and $95^{\circ} \mathrm{C}$ for $30 \mathrm{~s}$. The primers used are listed below: lamin B1, forward: $5^{\prime}$-TAGAGGAAAGCGGAAGAGGGTT-3' and reverse: 5'-CCAGATGAGGTCAGTTGGGGGG-3'; GAPDH, forward: 5'-AGGTCGGAGT-
CAACGGATTT-3' and reverse: 5'-TGACGGTGCCATGGAATTTG-3'.

Cell proliferation and colony formation assays. Two GC lines (SGC-7901 and MGC-803), were infected with negative control siRNA or with siRNA targeting lamin B1 and seeded in a plate of 96-well (density, 10000 cells in $200 \mu \mathrm{l} /$ well) and cultured at $5 \% \mathrm{CO}_{2}$ and $37^{\circ} \mathrm{C}$ in an incubator. An MTT assay was used to determine cell proliferation rates. Absorbance values were obtained at $490 \mathrm{~nm}$. The cells infected with siRNA targeting lamin B1 or with negative control siRNA were seeded in 6-well culture plates for the colony formation assay. After 14 days of incubation, cell colonies were fastened with $10 \%$ polytetrafluoroethylene (PFA) and dyed with crystal violet solution. Using a light microscope, we counted colonies with more than 50 cells.

Cell scratch and Transwell invasion assays. The cell scratch assay was used to determine the migration ability of GC cells. There were $3 \times 10^{5}$ cells seeded into 6-well culture plates. A Micro Scratch Tester (MST) was used to observe three parallel linear wounds generated in each plate. After culturing for $24 \mathrm{~h}$, we assessed the migration ability. For the measurement of the invasive ability, the Transwell invasion assay, which was matrix-coated, was conducted. Then, we transferred $1 \times 10^{5}$ suspended cells to the upper chamber of a matrix-coated 24-well plate from $500 \mu \mathrm{l}$ of cell culture medium, for $24 \mathrm{~h}$ incubation. Later, we fastened the membranes and stained them with a crystal violet solution for $10 \mathrm{~min}$. Finally, we calculated the sum of cells that invaded the membranes.

Cell adhesion test. We dried the Matrigel $(2 \mu \mathrm{g} / 50 \mu \mathrm{l})$ after adding it to a 96-well plate. The excess gel was washed away from the plate using a phosphate buffer solution (PBS). In a cell incubator of $37^{\circ} \mathrm{C}$, the logarithmic stage on a Matrigel plate was used to seed four thousand cells for $40 \mathrm{~min}$. Then, we removed the unadhered cells besides the culture solution. By using the Celigo image cytometer, the cell number was counted.

Western blot. In the RIPA lysis buffer, we extracted total protein from GC cells. We blotted equal amounts of protein onto the polyvinylidene fluoride membranes (Amersham, UK) after separating them by using $10 \%$ sodium dodecyl sulfonate polyacrylamide gel electrophoresis (SDS-PAGE). At room temperature, we blocked the membranes in 5\% bovine serum albumin (BSA) for $1 \mathrm{~h}$ and incubated them with primary antibodies overnight. For secondary HRP-conjugated antibodies, the incubation at room temperature lasted for $1 \mathrm{~h}$ (Perbio Science, Belgium). Using the ECL immunodetection system (Immobilon, USA), we visualized immunoreactive proteins. The antibodies, which were used for western blot, were as follows: anti-lamin B1 (12987-1-AP, Proteintech Group, USA), anti-PTEN (22034-1-AP, Proteintech Group, USA), anti-PI3 Kinase p85 (ab191606, Abcam, USA), antipan-AKT (ab38449, Abcam, USA), anti-AKT (10176-2-AP, Proteintech Group, USA), anti-Phospho-MEK1/2 (CST: \#9154, Proteintech Group, USA), anti-MEK (CST: \#8727, 
Cell Signaling Technology, USA), anti-Phospho-ERK1/2 (CST: \#4370, Proteintech Group, USA), anti-ERK (164431-AP, Proteintech Group, USA), anti-P53 (10442-1-AP, Proteintech Group, USA), anti-P21 (10355-1-AP, Proteintech Group, USA), and anti- $\beta$-actin (20536-1-AP, Proteintech Group, USA).

Immunohistochemistry (IHC) and scoring. We obtained permission from the Medical Ethics Committee of Lanzhou University Second Hospital and informed consent from all patients concerned. After washing the gastric tumor and paracarcinoma tissue sections (thickness, $4 \mu \mathrm{m}$ ) in xylene for two times (15 min each), we did the $10 \mathrm{~min}$ sequential washes as follows: a $50 \%$ ethanol $/ 50 \%$ xylene mixture; $75 \%$, $85 \%$, 95\%, and $100 \%$ ethanol; $3 \% \mathrm{H}_{2} \mathrm{O}_{2}$; and $\mathrm{ddH}_{2} \mathrm{O}$. After retrieving the antigen, we blocked the sections in $10 \%$ bovine serum albumin (BSA) for $30 \mathrm{~min}$. The incubation proceeded in a humidified chamber with anti-lamin B1 (12987-1-AP, Proteintech Group, USA) at $4{ }^{\circ} \mathrm{C}$ overnight. Then, using an UltraSensitiveTM SP kit (Fuzhou Maixin Biotech. Co., Ltd.) in the same chamber, we incubated them at room temperature for $1 \mathrm{~h}$. Therefore, the images from an inverted microscope were acquired after dyeing $\mathrm{DAB}$ and hematoxylin (XDS-100, Shanghai Cai Kang Optical Instrument Co., Ltd.).

Lamin B1 immunostaining was scored by two pathologists based on the dyeing strength and percentage of dyed cells in each section aforementioned [10]. The intensity score included: "0" (negative dyeing), "1" (weak dyeing), "2" (moderate dyeing), or " 3 " (strong dyeing). The percentage of positively dyed cells comprised " 0 " (0\%), “1” (1-25\%), “2” (26-50\%), “3” (51-75\%), “4” (76-100\%). Then, we obtained the final score by calculating the product of two scores. The median lamin B1 IHC score (6.0), was selected as the cut-off value to divide the samples into two groups: the high expression group and the low expression group.

\section{Results}

Lamin B1 expression was downregulated in GC tissues and associated with clinical stage, depth of invasion, nodal stage, and poor prognosis. To investigate whether lamin B1 plays a vital role in GC progression, we first examined the expression level of lamin B1 in GC tissue by immunochemical staining. We found that lamin B1 is significantly decreased in GC tissues than paracarcinoma tissues (Figure 1A). Next, we investigated the relationship between clinicopathological features and the lamin B1 level in GC. Our data showed that low level of lamin B1 in GC was significantly positive correlated with higher clinical stage $(p=0.01$, Figure $1 B)$, depth of invasion $(\mathrm{p}=0.001)$, nodal stage $(\mathrm{p}=0.005)$, and poor prognosis. However, there were no significant and promi-
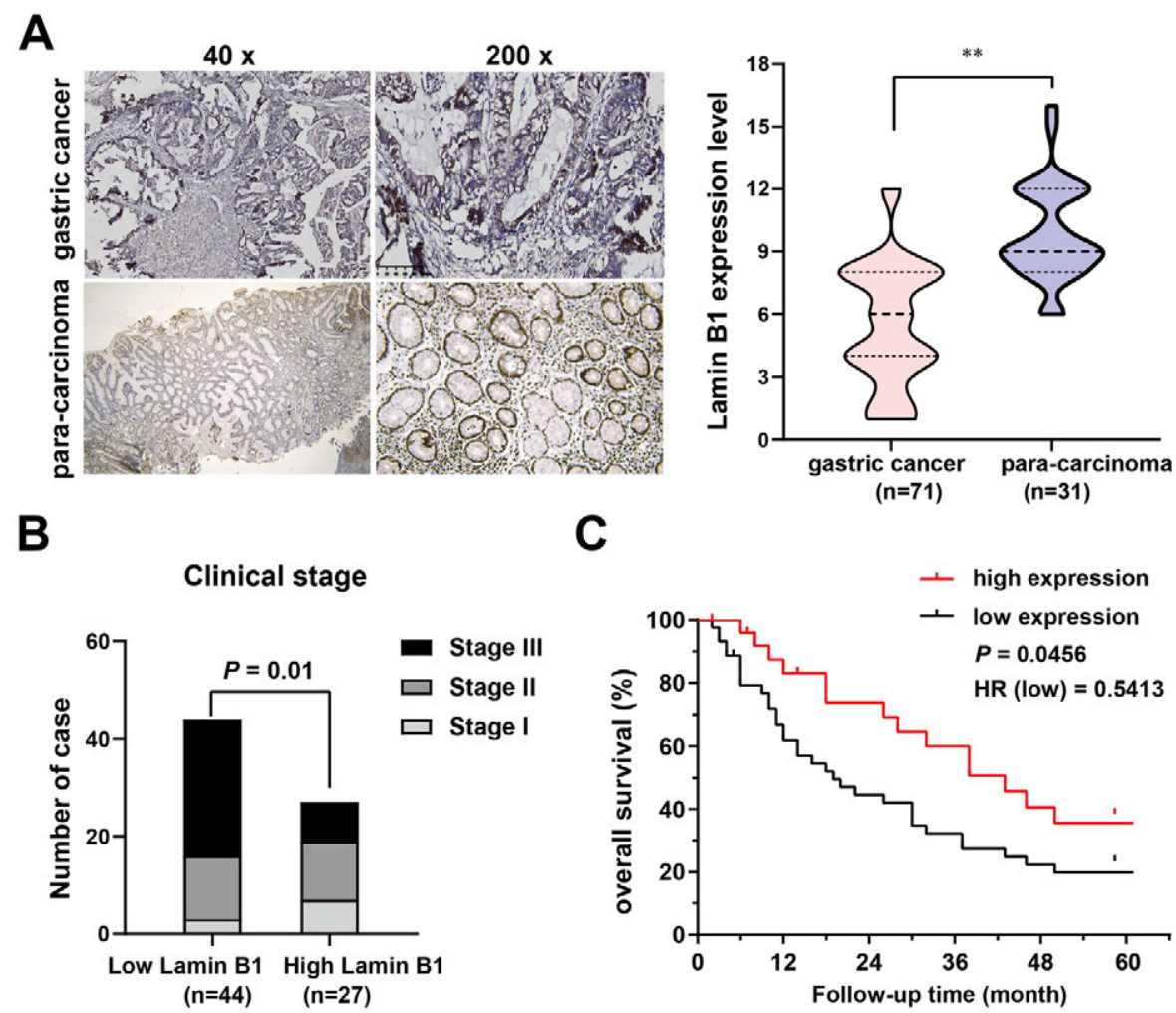

C

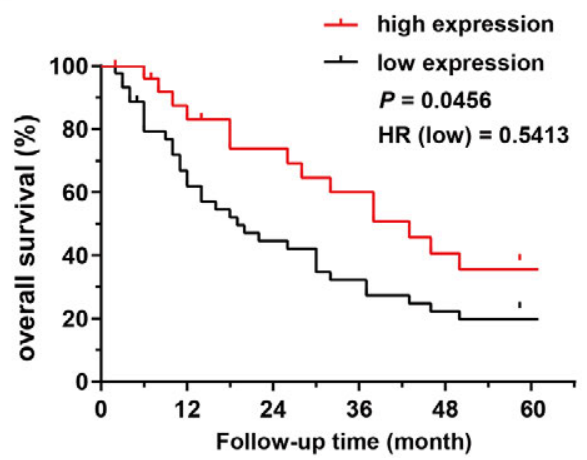

Figure 1. Expression of lamin B1 and its association with prognosis in gastric cancer patients. A) Representative images of immunohistochemical staining for lamin B1 in gastric cancer tissues and paracarcinoma tissues; t-test was used to examine statistical significance. B) Correlation between lamin B1 expression and the clinicopathologic characteristics of gastric cancer patients; the chi-square test was used to examine statistical significance. C) The Kaplan-Meier survival analysis of patients with lamin B1-low $(\mathbf{n}=44)$ or lamin B1-high $(\mathbf{n}=27)$ group. ${ }^{* *} \mathbf{p}<0.01$ 
nent differences observed for differentiation degree, age, sex, or Lauren classification (Table 1). Kaplan-Meier analyses revealed that the overall survival rate of the lamin B1-high patients was markedly better than that of the lamin B1-low patients (Figure 1C). Furthermore, the multivariable hazard ratios for variables were computed using a Cox regression model. It turned out that lamin B1 was an independent factor of prognosis in GC (Table 2). To sum up, our results showed that reduced expression of lamin $\mathrm{B} 1$ predicts poor prognosis in patients diagnosed with GC.

Table 1. Relationships between lamin B1 expression and clinicopathological characteristics in gastric cancer patients.

\begin{tabular}{|c|c|c|c|}
\hline Variables & $\begin{array}{c}\text { High } \\
\text { expression } \\
(\mathbf{n}=27)\end{array}$ & $\begin{array}{c}\text { Low } \\
\text { expression } \\
(\mathrm{n}=44)\end{array}$ & p-value \\
\hline Age & $55.89 \pm 11.55$ & $56.66 \pm 12.82$ & 0.799 \\
\hline \multicolumn{4}{|l|}{ Sex } \\
\hline male & $18(66.7 \%)$ & $31(70.5 \%)$ & \multirow{2}{*}{0.738} \\
\hline female & $9(33.3 \%)$ & $13(29.5 \%)$ & \\
\hline \multicolumn{4}{|l|}{ Differentiation degree } \\
\hline poorly differentiated & $14(51.9 \%)$ & $32(72.7 \%)$ & \multirow{3}{*}{0.146} \\
\hline moderately differentiated & $10(37.0 \%)$ & $10(22.7 \%)$ & \\
\hline highly differentiated & $3(11.1 \%)$ & $2(4.6 \%)$ & \\
\hline \multicolumn{4}{|l|}{ Histological type } \\
\hline intestinal & $23(85.2 \%)$ & $31(70.5 \%)$ & \multirow{2}{*}{0.158} \\
\hline diffuse & $4(14.8 \%)$ & $13(29.5 \%)$ & \\
\hline \multicolumn{4}{|l|}{ Stage of disease } \\
\hline I & $7(25.9 \%)$ & $3(6.8 \%)$ & \multirow{3}{*}{0.01} \\
\hline II & $12(44.5 \%)$ & $13(29.6 \%)$ & \\
\hline III & $8(29.6 \%)$ & $28(63.6 \%)$ & \\
\hline \multicolumn{4}{|l|}{ Clinical tumor stage } \\
\hline $\mathrm{T} 1$ & $9(33.3 \%)$ & $2(4.6 \%)$ & \multirow{4}{*}{0.001} \\
\hline $\mathrm{T} 2$ & $13(48.2 \%)$ & $14(31.8 \%)$ & \\
\hline $\mathrm{T} 3$ & $4(14.8 \%)$ & $21(47.7 \%)$ & \\
\hline $\mathrm{T} 4$ & $1(3.7)$ & $7(15.9 \%)$ & \\
\hline \multicolumn{4}{|l|}{ Clinical nodal stage } \\
\hline No & $9(33.3 \%)$ & $2(4.6 \%)$ & \multirow{4}{*}{0.005} \\
\hline N1 & $7(25.9 \%)$ & $13(29.5 \%)$ & \\
\hline $\mathrm{N} 2$ & $10(37.0 \%)$ & $20(45.4 \%)$ & \\
\hline $\mathrm{N} 3$ & $1(3.7 \%)$ & $9(20.5 \%)$ & \\
\hline
\end{tabular}

Table 2. Multivariate analysis of survival in gastric cancer patients.

\begin{tabular}{lcc}
\hline Variables & $\begin{array}{c}\text { Hazard ratio } \\
(\mathbf{9 5 \%} \mathbf{C I})\end{array}$ & p-value \\
\hline Lamin B1 expression & $1.848(1.039-3.287)$ & 0.0456 \\
Age & $1.014(0.981-1.047)$ & 0.405 \\
Sex & $0.95(0.396-2.276)$ & 0.909 \\
Differentiation degree & $0.62(0.261-1.475)$ & 0.28 \\
Histological type & $0.797(0.354-1.793)$ & 0.583 \\
Stage of disease & $26.481(6.093-115.098)$ & $<0.001$ \\
Clinical tumor stage & $9.252(2.646-32.349)$ & $<0.001$ \\
Clinical nodal stage & $17.082(4.702-62.062)$ & $<0.001$ \\
\hline
\end{tabular}

Suppression of lamin B1 promoted gastric cancer cell proliferation and migration. To investigate how lamin $\mathrm{B} 1$ regulates GC progression, we used lamin B1 siRNA to knock down lamin B1 expression in MGC-803 and SGC-7901 cells. After $48 \mathrm{~h}$, we examine the transfection efficiency by western blotting and qRT-PCR. Our data showed that the expression of lamin B1 was dramatically decreased in MGC-803 and SGC-7901 cells (Figures 2A, 2B). Moreover, as shown in Figure 2C, after inhibiting lamin B1 expression using lamin B1 siRNA, the MTT assay showed that the proliferation of MGC-803 and SGC-7901 GC cells was significantly increased. We further proved the impact of lamin B1 on cell growth by using colony formation assays. As shown in Figure 3D, the suppression of lamin $\mathrm{B} 1$ promoted the colony formation of GC cells. These results indicated that the suppression of lamin B1 expression promoted GC cell proliferation. Then, we performed cell scratch assay, Transwell assays, and cell adhesion assays to evaluate the effects of lamin B1 knockdown on the migration of GC cells. As shown in Figures 3A and 3B, the migration rates of MGC-803 and SGC-7901 cells transfected with siRNA-lamin B1 were significantly increased at $24 \mathrm{~h}$ in comparison with those of the corresponding control cells, as measured by the cell scratch assay. Moreover, the difference was found in the adhesion rate of cells between the siRNA-lamin B1 and control samples in the adhesion test in MGC-803 cells. (Figure 3C). Matrix-coated Transwell assays also showed that GC cell migration ability greatly increased in MGC-803 and SGC-7901 cells transfected with siRNAlamin B1 in comparison with control groups (Figure 3D).

Overexpression of lamin B1 suppresses gastric cancer cell proliferation and migration. Next, we investigate how overexpression of lamin B1 affects GC cell proliferation and migration.

As shown in Figures $4 \mathrm{~A}$ and $4 \mathrm{~B}$, we firstly confirmed the transfection efficiency by qRT-PCR and western blotting. Our data further showed that the overexpression of lamin B1 could inhibit the proliferation of MGC-803 and SGC-7901 GC cells by MTT assay and colony formation assays (Figures 4C, 4D). Moreover, using cell scratch assays (Figures 5A, 5B), Transwell assays (Figure 5C), and cell adhesion assays (Figure 5D), we also further confirmed that the overexpression of lamin B1 could significantly suppress GC cell migration.

Lamin B1 deficiency promoted GC progression by decreasing the p53/p21 $1^{\mathrm{WAF} 1 / \mathrm{CIP} 1}$ expression and activating the MAPK/ERK and PI3K/PTEN/Akt pathways. The underlying mechanism of how lamin B1 deficiency promoted GC progression was explored next. Our data indicated that the p53 and p21 expressions were remarkably downregulated in MGC-803 and SGC-7901 cells with lamin B1 silencing (Figure 6A), in comparison with the lamin B1 overexpression group (Figure 6B). Follow-up studies indicated that the lamin B1 deficiency increased the levels of one subunit of PI3K, p85a, and decreased the levels of the PTEN protein in MGC-803 and SGC-7901 cells. In these two cell lines, the 
level of phosphorylated Akt protein increased after knockdown of lamin B1. The activity of the MAPK/ERK pathway, represented by the levels of phosphorylated MEK and ERK proteins, was affected in both cell lines. The increased regulation of phospho-MEK and ERK levels after knockdown of lamin B1 was also found via a western blot assay. However, the total protein levels of PI3K, AKT, MEK, and ERK were minimally affected (Figure 6A). Contrary to the above results (Figure 6B), the overexpression of lamin B1 could suppress GC progression by increasing the p53/p21 $21^{\mathrm{WAF} 1 / \mathrm{CIP} 1}$ expression and inhibiting the MAPK/ERK and PI3K/PTEN/Akt pathways activation. These data suggest that lamin B1 homeostasis disorder plays a very important role in promoting GC progression.
A

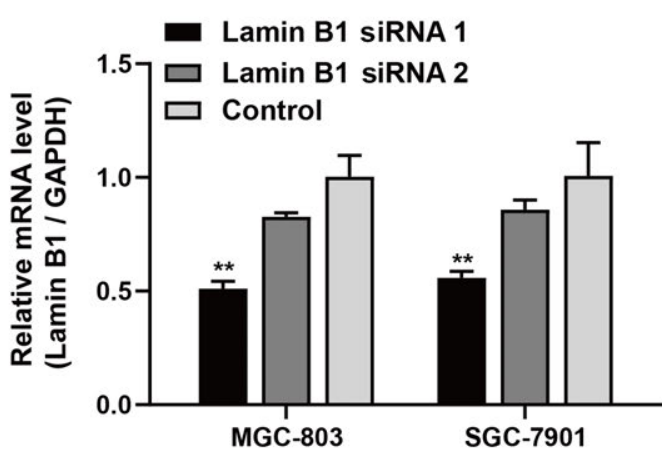

C

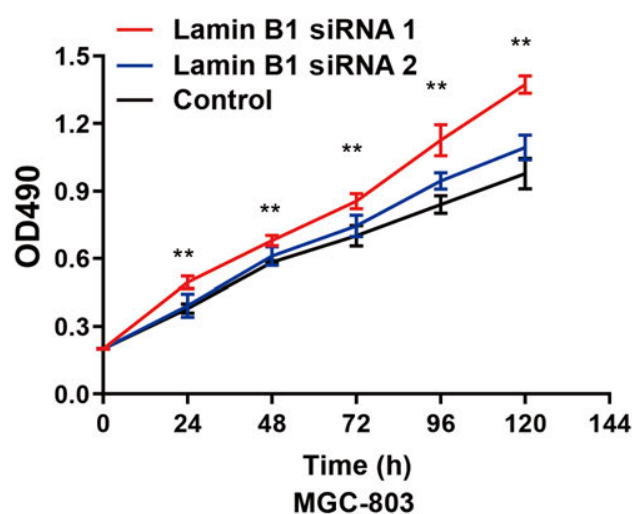

D

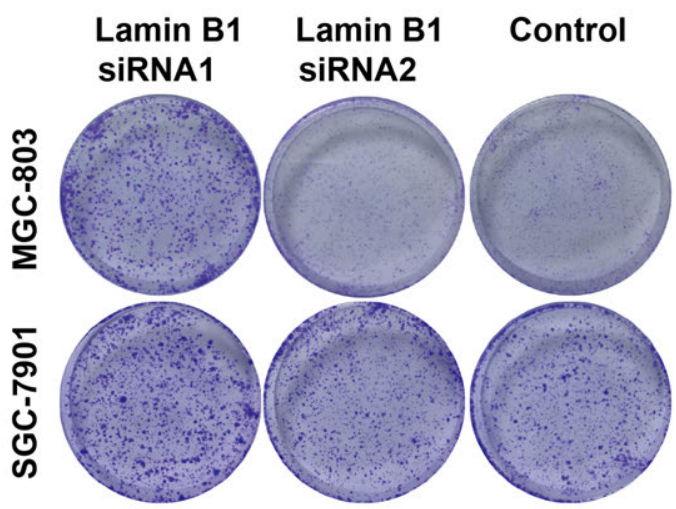

B
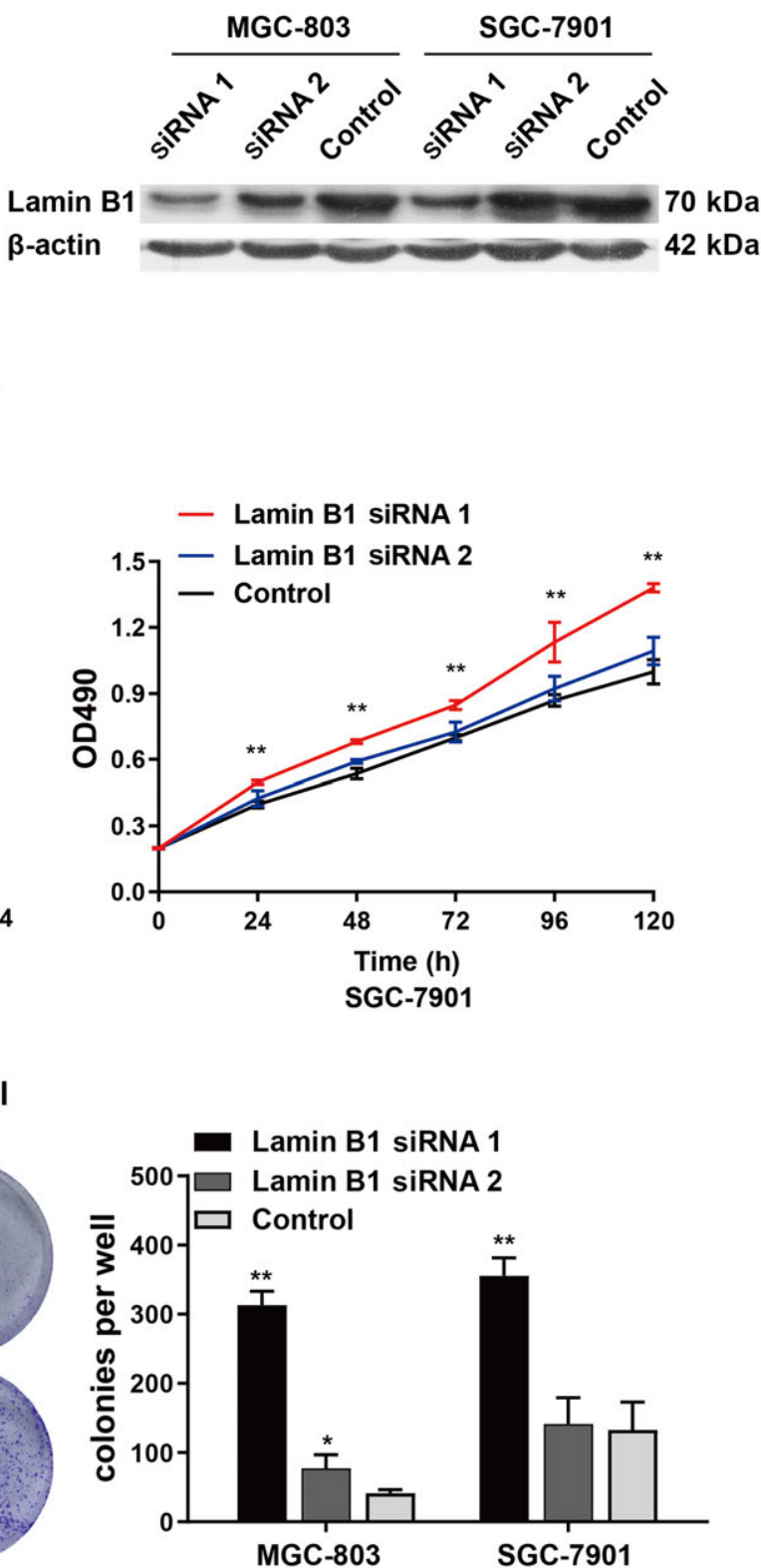

Figure 2. Suppression of lamin B1 promoted gastric cancer cell proliferation A) lamin B1 knockdown efficiencies were determined by RT-PCR; B) lamin B1 knockdown efficiencies were determined by western blotting. $\beta$-actin was used as an internal control. C) Proliferation ability was detected by MTT assays. D) Proliferation ability was detected by the colony-formation assay. One-way ANOVA was used to examine statistical significance. ${ }^{*} \mathrm{p}<0.05,{ }^{* *} \mathrm{p}<0.01$ 

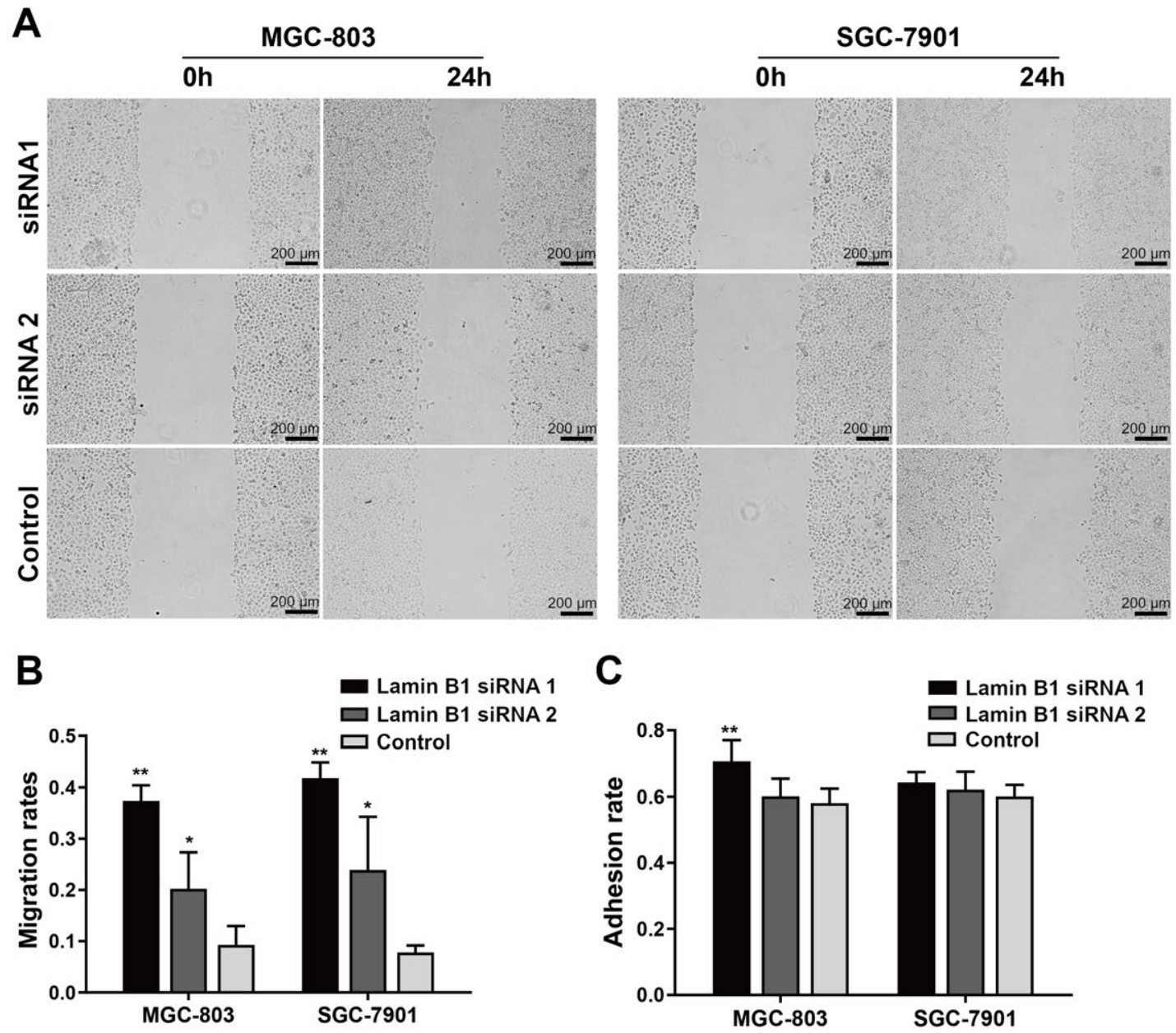

D
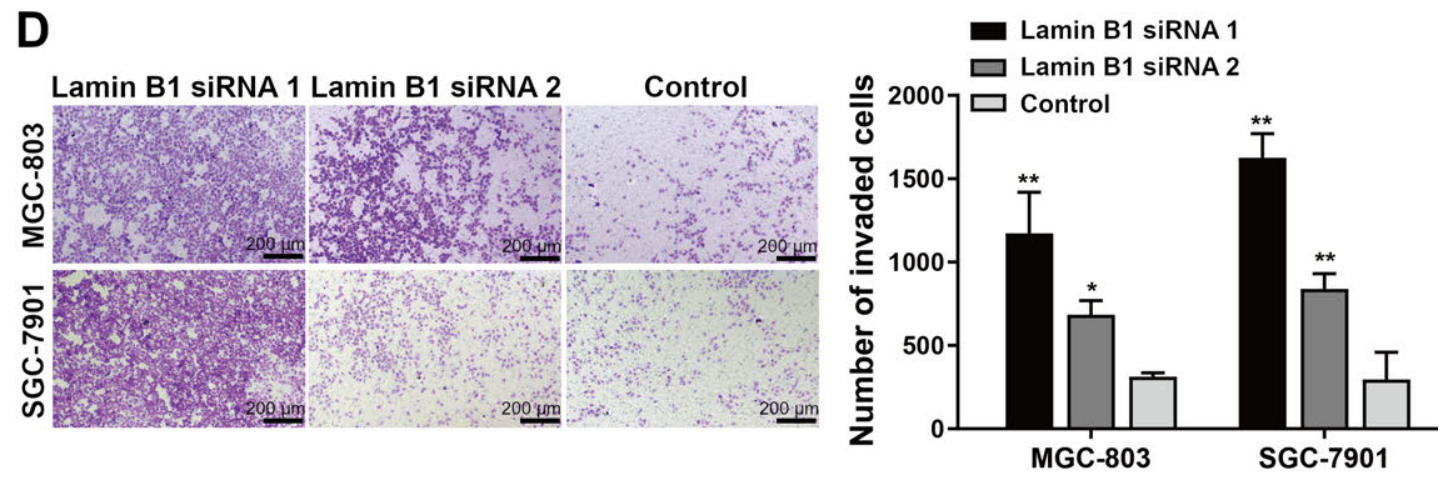

Figure 3. Suppression of lamin B1 promoted gastric cancer cell migration. A, B) Migration ability was detected by the cell scratch assay. C) A higher adhesion rate was found in the siRNA1 group of MGC-803 cells. D) Migration ability was detected by matrix-coated Transwell assay. One-way ANOVA was used to examine statistical significance. ${ }^{\star} \mathrm{p}<0.05,{ }^{* *} \mathrm{p}<0.01$

\section{Discussion}

As one of the nuclear matrix components, the nuclear envelope structural lamins are essential proteins of the nuclear lamina, involved in a series of fundamental cellular functions. Currently, they are indispensable proteins for the survival of mammalian somatic cells $[11,12]$. Lamins exist in the nuclear neighboring region between the inner nuclear and membrane chromatin and interact with these elements. In recent decades, lamins have been considered to maintain the shape of the nucleus and mechanical stability [13]. Nevertheless, recent studies suggest that they also play a vital role in 
A

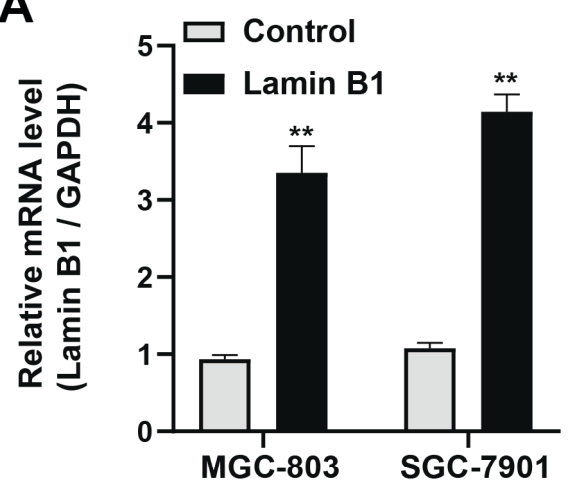

C

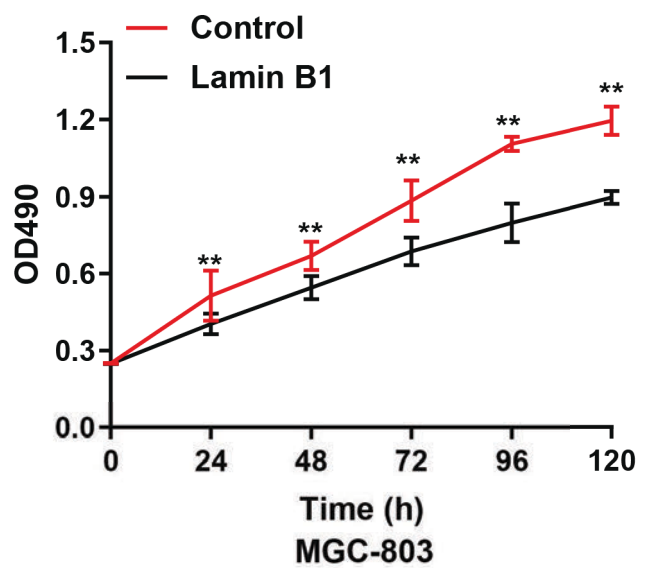

D

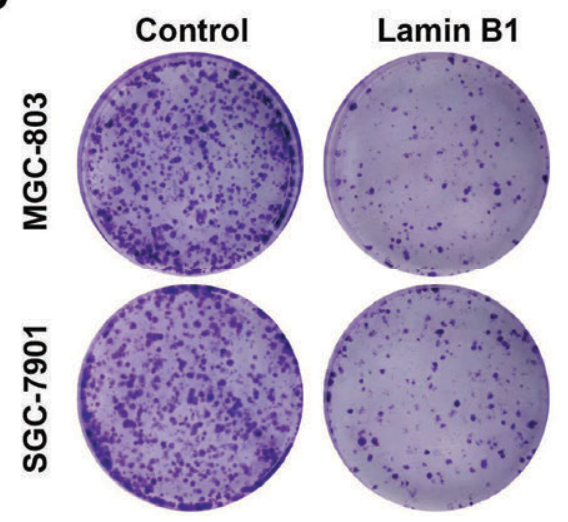

B

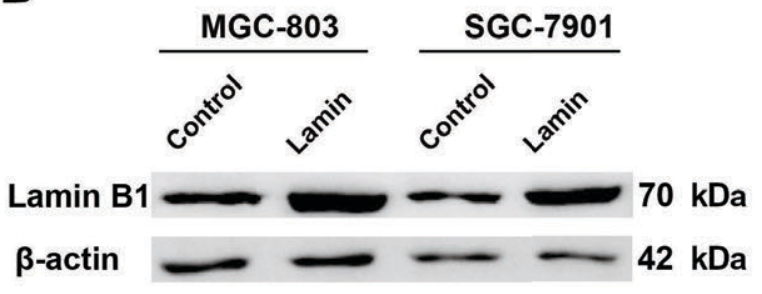

Figure 4. Overexpression of lamin B1 suppresses gastric cancer cell proliferation A) lamin B1 overexpression efficiencies were determined by RT-PCR; B) lamin B1 overexpression efficiencies were determined by western blotting. $\beta$-actin was used as an internal control. C) Proliferation ability was detected by MTT assays in MGC-803 and SGC-7901 with the lamin B1 overexpression. D) Proliferation ability was detected by the colony-formation assay in MGC-803 and SGC-7901 with lamin B1 overexpression. Student's $t$-test was used to examine statistical significance. ${ }^{\star} \mathbf{p}<0.05,{ }^{\star *} \mathrm{p}<0.01$

crucial cellular processes, such as replication of DNA, tumorigenesis, progression of the cell cycle, mitotic spindle formation, aging, differentiation of adult stem cell, and chromatin organization $[10,14-16]$. Studies on the lamins family have long focused on type A lamins, but few have focused on type B lamins, especially lamin B1. Some studies have suggested that the expression of multiple factors involved in replication and repair of DNA is dysregulated when lamin B1 levels are changed. Hence, lamin B1 is involved in a variety of cellular activities, and its abnormal expression is connected with tumor growth $[17,18]$. Nevertheless, the role of lamin B1 in the development and prognosis of cancer needs to be clari- 
A
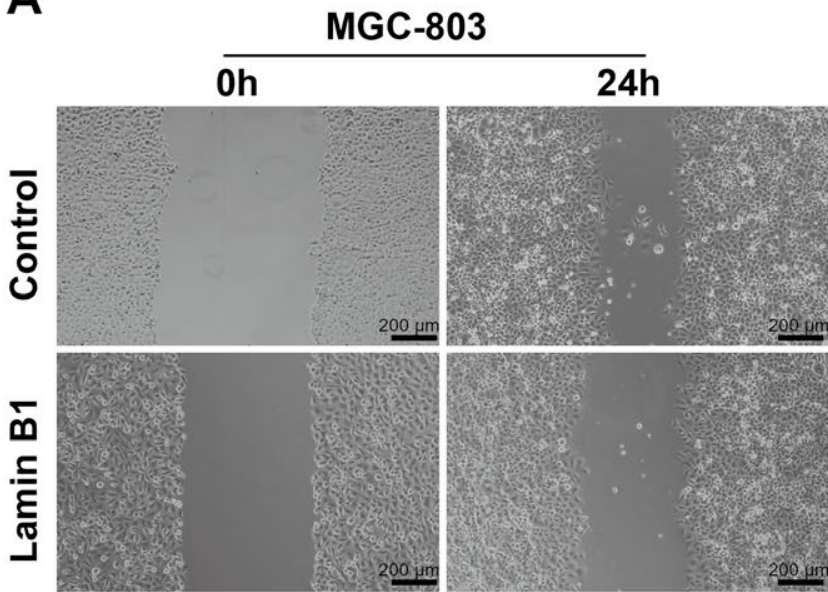

B

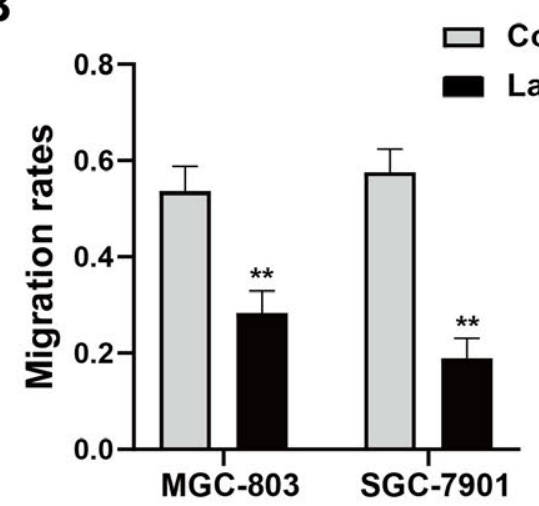

24h
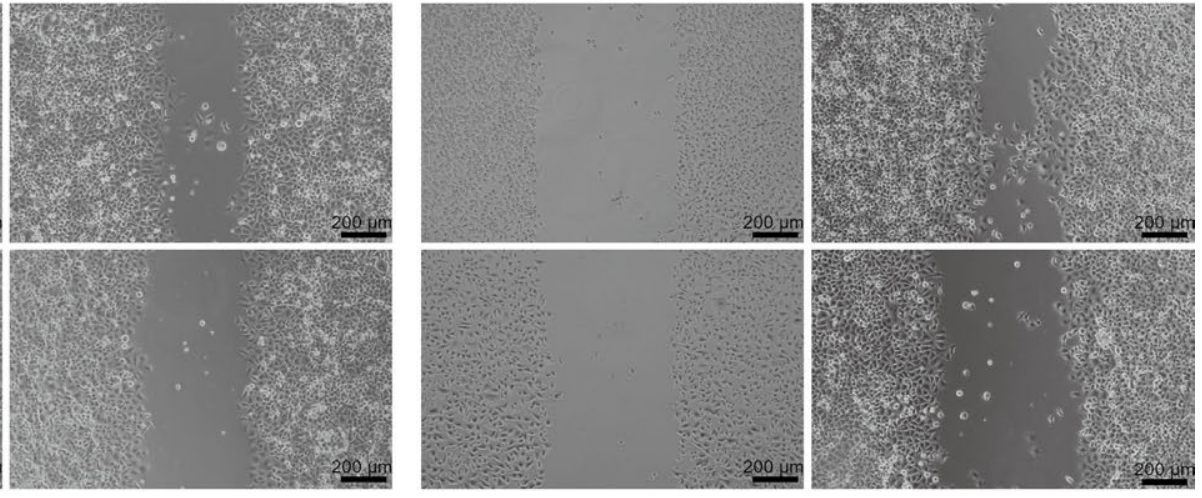

C

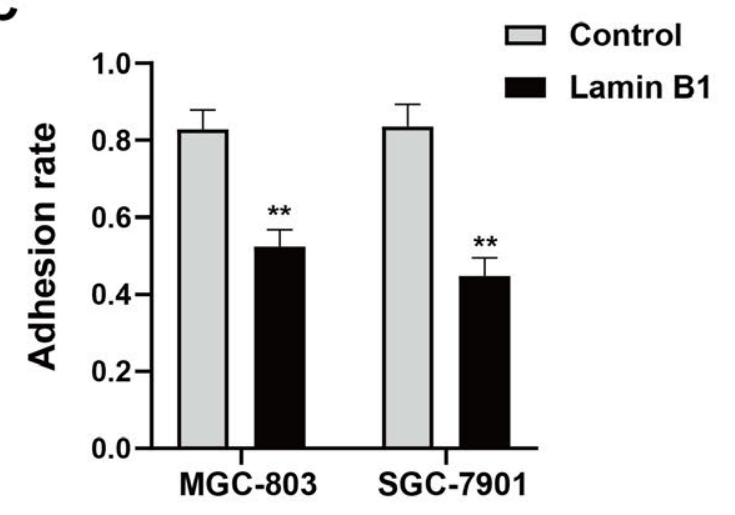

D
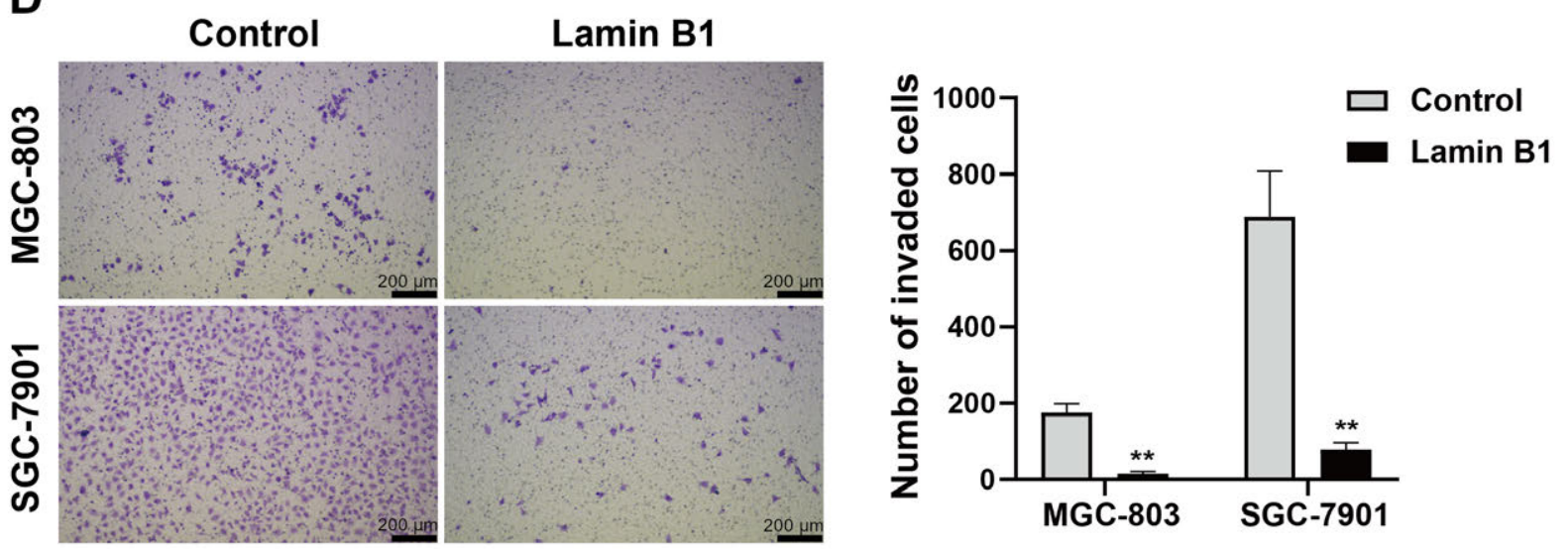

Figure 5. Overexpression of lamin B1 suppresses gastric cancer cell migration. A, B) Migration ability was detected by cell scratch assay in MGC-803 and SGC-7901 with lamin B1 overexpression. C) Migration ability was detected by the cell adhesion rate. D) Migration ability was detected by matrixcoated Transwell assay in MGC-803 and SGC-7901 with lamin B1 overexpression. Student's t-test was used to examine statistical significance. ${ }^{\star} \mathrm{p}<0.05$, ${ }^{\star *} \mathbf{p}<\mathbf{0 . 0 1}$

fied. Prior studies have also demonstrated diverse lamin B1 expression levels in different cancers. It is known that the lamin B1 expression is downregulated in colon cancer, breast cancer, and lung cancer [19-21]. While upregulated lamin B1 expression has been observed in pancreatic cancer and hepatocellular carcinoma prostate cancer [22-24]. Nevertheless, there are few systematic studies on lamin B1 in GC.

In this study, our IHC dyeing analysis showed a decrease in lamin B1 protein levels in GC tissues in comparison with that in paracarcinoma tissues. We found that reduced 

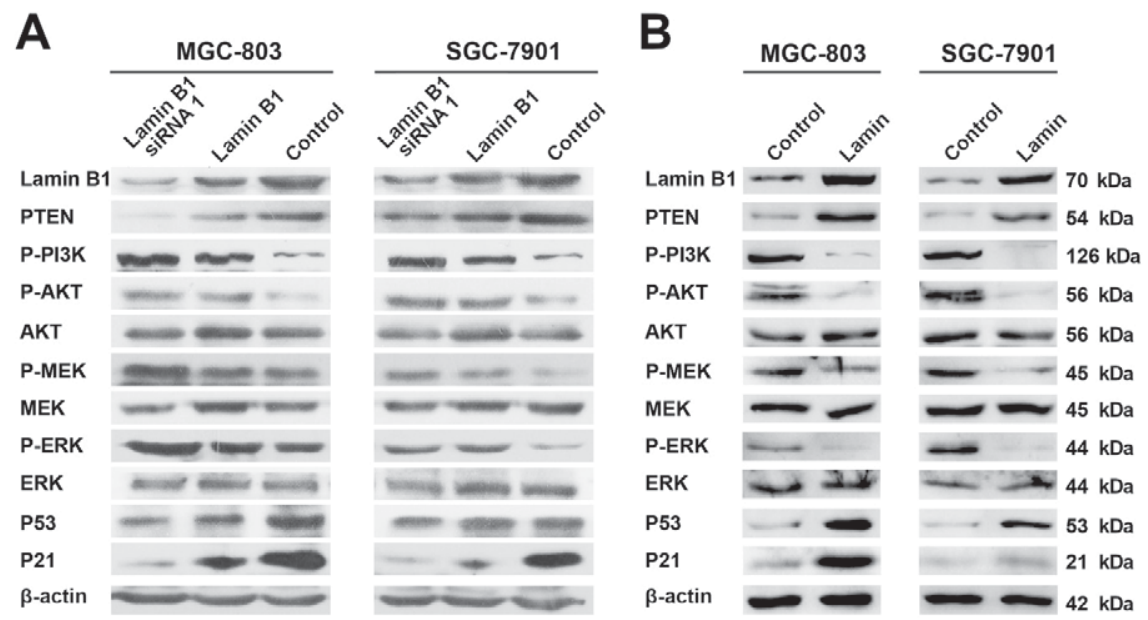

Figure 6. The effect of lamin B1 on p53/p21WAF1/CIP1 expression and the MAPK/ERK plus PI3K/PTEN/Akt pathways. A) The levels of MAPK/ERK and PI3K/PTEN/Akt pathways related proteins p53 and p21 were determined by western blotting in MGC-803 and SGC-7901 with lamin B1 knockdown. B) The levels of MAPK/ERK and PI3K/PTEN/Akt pathways related proteins p53 and p21 were determined by western blotting in MGC-803 and SGC-7901 with lamin B1 overexpression.

lamin B1 expression has a correlation with higher clinical stage, depth of invasion, nodal stage, and poor prognosis. In addition, stratified survival analyses suggested that the GC patients with higher levels in lamin B1 had better prognoses than those with lower levels in lamin B1, implying that lamin B1 could be an independent prognosis factor in GC. As previously shown in lung cancer [19], lamin B1 as a tumor suppressor gene also plays an important role in GC progression. However, the effect of lamin B1 deficiency on GC cells is unknown. Moreover, the biological function of lamin B1 is closely related to cell proliferation, and its high expression may be closely related to changes in cell growth characteristics and potential tumorigenic potential. Therefore, we further investigate how lamin B1 disorder functions in GC cells. To research into the regulatory effect of lamin B1 in GC cells, we then investigated how the expression of lamin B1 could have a significant impact on GC proliferative and metastatic abilities. As expected, we observed that lamin B1 disorder is associated with GC proliferation and migration.

Nonhomologous end joining (NHEJ) and homologous recombination repair (HRR), which are the main mechanisms of DSB repair, are drastically impaired in cells with downregulated lamin B1 levels, leading to persistent DNA damage signaling. Meantime, previous studies showed that deficiency of LMNB1 triggers H3K27me3 toward the interior of the nucleus and resulted in enlarged nuclear speckles, thereby increasing genomic instability [25]. Moreover, lamin B1 silence could lead to the loss of the polycomb repressive complex 2 (PRC2) recruitment, thereby activating the $\mathrm{RET} / \mathrm{p} 38$ signaling axis to promote cancer progression [19]. Previous studies have also proved that p53 is a key factor in modulating repair and recombination of DNA through HRR and NHEJ [26, 27]. Thus, we hypothesize that the downregulation of lamin B1 probably has a profound effect on the p53 gene. To clarify the relationship between lamin $\mathrm{B} 1$ and the p53 gene, we next investigated whether lamin B1 exerted any influence on the signaling pathway of p53 in GC. The analysis results of western blot revealed that lamin B1 appreciably affected the protein levels of p53 and its downstream effector p21. The signaling pathway of ERK-MAPK has a connection with the malignant development of diverse cancers [28-33]. Furthermore, ERK/MAPK was shown to be related to lamin B1 in spatial learning [34]. Thus, we performed western blot analysis to examine whether reduced or increased lamin B1 expression regulates the activation of ERK/MAPK. Our results revealed that lamin B1 deficiency promoted GC cell growth and metastasis partly because of the ERK/MAPK pathway activation. The PI3K/Akt signaling pathway is of great significance in cell proliferation, migration, invasion, and cell cycle progression in human gastric cancer [35]. Moreover, it has been recorded that in prostate cancer, PI3K/ $\mathrm{AKT}$ is regulated by lamin A/C [36]. Therefore, considering the significance of the PI3K/Akt signaling pathway in the tumor development and its interaction with lamin A/C, we next investigated whether lamin $\mathrm{B} 1$ exerted an extremely important influence on the pathway of PI3K/Akt in GC. We performed western blot to examine the influences of lamin B1 on the p85a, Akt, and p-Akt protein levels. Our data indicated that knockdown of lamin B1 noticeably increased the protein levels of $\mathrm{p} 85 \mathrm{a}$ and $\mathrm{p}$-Akt by reducing the protein level of PTEN, in comparison with the result of lamin B1 overexpression.

In conclusion, we demonstrated that lamin B1 was remarkably downregulated in GC tissues and reduced expression of lamin B1 predicts poor prognosis for GC patients. Lamin B1 homeostasis disorder plays a very important role in promoting GC proliferation and migration in vitro. More importantly, lamin B1 deficiency in GC could activate 
two pathways of ERK/MAPK and PI3K/PTEN/Akt, with a reduction in $\mathrm{p} 53 / \mathrm{p} 21^{\mathrm{WAF} 1 / \mathrm{CIP} 1}$ expression. Therefore, lamin B1 might act as a tumor inhibitory protein that suppresses the proliferation and metastasis of GC cells. Lamin B1 could be used as a marker for molecules in the development of GC as well as a potential therapeutic target in the future.

Acknowledgments: This research was funded by the Fundamental Research Funds for the Central Universities (lzujbky-2019-cd06) and Lanzhou Chengguan District Science and Technology Bureau Program (No. 2019SHFZ0012) and Lanzhou University Second Hospital Cuiying Program (No. CY2018-QN11).

\section{References}

[1] BRAY F, FERLAY J, SOERJOMATARAM I, SIEGEL RL, TORRE LA et al. Global cancer statistics 2018: globocan estimates of incidence and mortality worldwide for 36 cancers in 185 countries. CA Cancer J Clin 2018; 68: 394-424. https:// doi.org/10.3322/caac. 21492

[2] LAZAR DC, TABAN S, CORNIANU M, FAUR A, GOLDIŞ A et al. New advances in targeted gastric cancer treatment. World J Gastroenterol 2016; 22: 6776-6799. https://doi. org/10.3748/wjg.v22.i30.6776

[3] BRAY F, FERLAY J, SOERJOMATARAM I, SIEGEL RL, TORRE LA et al. Global cancer statistics 2018: globocan estimates of incidence and mortality worldwide for 36 cancers in 185 countries. CA Cancer J Clin 2018; 68: 394-424. https:// doi.org/10.3322/caac. 21492

[4] TER VEER E, MOHAMMAD NH, LODDER P, NGAI LL, SAMAAN M et al. The efficacy and safety of S-1-based regimens in the first-line treatment of advanced gastric cancer: a systematic review and meta-analysis. Gastric Cancer 2016; 19: 696-712. https://doi.org/10.1007/s10120-015-0587-8

[5] GUELEN L, PAGIE L, BRASSET E, FAZA MB, TALHOUTET W et al. Domain organization of human chromosomes revealed by mapping of nuclear Lamina interactions. Nature 2008; 453: 948-951. https://doi.org/10.1038/nature06947

[6] MALHAS A, LEE F, SANDERS R, SAUNDERS NJ, VAUX DJ. Defects in Lamin B1 Expression or Processing Affect Interphase Chromosome Position and Gene Expression. J Cell Biol 2007; 176: 593-603. https://doi.org/10.1083/ jcb.200607054

[7] BUTIN-ISRAELI V, ADAM SA, JAIN N, OTTE GL, NEEMS D et al. Role of Lamin B1 in Chromatin Instability. Mol Cell Biol 2015; 35: 884-898. https://doi.org/10.1128/ MCB.01145-14

[8] DREESEN O, ONG PF, CHOJNOWSKI A, COLMAN A. The contrasting roles of Lamin B1 in cellular aging and human disease. Nucleus 2013; 4: 283-290. https://doi. org/10.4161/nucl.25808

[9] WAZIR U, MAI HA, BRIDGER JM, HARVEY A, JIANG WG et al. The clinicopathological significance of Lamin A/C, Lamin B1 and Lamin B receptor mRNA expression in human breast cancer. Cell Mol Biol Lett 2013; 18: 595-611. https://doi.org/10.2478/s11658-013-0109-9
[10] AO Y, ZHANG J, LIU Z, QIAN M, LI Y et al. Lamin A buffers CK2 kinase activity to modulate aging in a progeria mouse model. Sci Adv 2019; 5: eaav5078. https://doi.org/10.1126/ sciadv.aav5078

[11] HARBORTH J, ELBASHIR SM, BECHERT K, TUSCHL T, WEBER K. Identification of essential genes in cultured mammalian cells using small interfering RNAs. J Cell Sci 2001; 114: 4557-4565

[12] TURGAY Y, EIBAUER M, GOLDMAN AE, SHIMI T, KHAYAT $\mathrm{M}$ et al. The molecular architecture of Lamins in somatic cells. Nature 2017; 543: 261-264. https://doi. org/10.1038/nature21382

[13] DITTMER TA, MISTELI T. The Lamin protein family. Genome Biol 2011; 12: 222. https://doi.org/10.1186/gb-201112-5-222

[14] YOON MH, KANG SM, LEE SJ, WOO TG, OH AY et al. p53 induces senescence through Lamin A/C stabilizationmediated nuclear deformation. Cell Death Dis 2019; 10: 107. https://doi.org/10.1038/s41419-019-1378-7

[15] PROKOCIMER M, DAVIDOVICH M, NISSIM-RAFINIA M, WIESEL-MOTIUK N, BAR DZ et al. Nuclear Lamins: key regulators of nuclear structure and activities. J Cell Mol Med 2009; 13: 1059-1085. https://doi.org/10.1111/j.15824934.2008.00676.x

[16] CAMOZZI D, CAPANNI C, CENNI V, MATTIOLI E, COLUMBARO $M$ et al. Diverse Lamin-dependent mechanisms interact to control chromatin dynamics: Focus on Laminopathies. Nucleus 2014; 5: 427-440. https://doi.org/10.4161/ nucl.36289

[17] BUTIN-ISRAELI V, ADAM SA, GOLDMAN RD. Regulation of nucleotide excision repair by nuclear Lamin b1. PLoS One 2013; 8: e69169. https://doi.org/10.1371/journal. pone.0069169

[18] TANG CW, MAYAMENDOZA A, MARTIN C, ZENG K, CHEN S et al. The integrity of a Lamin-B1-dependent nucleoskeleton is a fundamental determinant of RNA synthesis in human cells. J Cell Sci 2008; 121: 1014-1024. https://doi. org/10.1242/jcs.020982

[19] JIA Y, VONG JS, ASAFOVA A, GARVALOV BK, CAPUTO $\mathrm{L}$ et al. Lamin B1 loss promotes lung cancer development and metastasis by epigenetic derepression of RET. J Exp Med 2019; 216: 1377-1395. https://doi.org/10.1084/jem.20181394

[20] IZDEBSKA M, GAGAT M, GRZANKA A. Overexpression of Lamin B1 induces mitotic catastrophe in colon cancer LoVo cells and is associated with worse clinical outcomes. Int J Oncol 2018; 52: 89-102. https://doi.org/10.3892/ ijo.2017.4182

[21] WAZIR U, AHMAD MH, BRIDGER JM, HARVEY A, JIANG WG et al. The clinicopathological significance of Lamin A/C, Lamin B1 and Lamin B receptor mRNA expression in human breast cancer. Cell Mol Biol Lett 2013; 18: 595-611. https://doi.org/10.2478/s11658-013-0109-9

[22] LI L, DU Y, KONG X, LI Z, JIA Z et al. Lamin B1 Is a Novel Therapeutic Target of Betulinic Acid in Pancreatic Cancer. Clin Cancer Res 2013; 19: 4651-4661. https://doi. org/10.1158/1078-0432.CCR-12-3630 
[23] SAARINEN I, MIRTTI T, SEIKKULA H, BOSTRÖM PJ, TAIMEN P et al. Differential Predictive Roles of A- and BType Nuclear Lamins in Prostate Cancer Progression. PLoS One 2015; 10: e0140671. https://doi.org/10.1371/journal. pone. 0140671

[24] IDRISS NK, FAKHRY M, IMAM HM, ABD-ELMOEZ FA, ABDELEWAHAB $\mathrm{H}$ et al. Analysis of Lamin B1, Vimentin and Anti-Ku86 as Prospective Biomarkers of Hepatocellular Carcinoma in Patients with Hepatitis C Virus Infection. Cell Physiol Biochem 2019; 52: 595-605. https://doi. org/10.33594/000000042

[25] CAMPS J, ERDOS MR, RIED T. The role of Lamin B1 for the maintenance of nuclear structure and function. Nucleus 2015; 6: 8-14. https://doi.org/10.1080/19491034.2014.10035 10

[26] MELLO SS, ATTARDI LD. Deciphering p53 signaling in tumor suppression. Curr Opin Cell Biol 2018; 51: 65-72. https://doi.org/10.1016/j.ceb.2017.11.005

[27] ARIAS-LOPEZ C, LAZARO-TRUEBA I, KERR P, LORD CJ, DEXTER T et al. p53 modulates homologous recombination by transcriptional regulation of the RAD51 gene. EMBO Rep 2006; 7: 219-224. https://doi.org/10.1038/ sj.embor.7400587

[28] WU S, LAO XY, SUN TT, REN LL, KONG X et al. Knockdown of ZFX inhibits gastric cancer cell growth in vitro and in vivo via downregulating the ERK-MAPK pathway. Cancer Lett 2013; 337: 293-300. https://doi.org/10.1016/j.canlet.2013.04.003

[29] LIU L, ZHANG H, SUN L, GAO Y, JIN H et al. ERK/MAPK activation involves hypoxia-induced MGr1-Ag/37LRP expression and contributes to apoptosis resistance in gastric cancer. Int J Cancer 2010; 127: 820-829. https://doi. org/10.1002/ijc.25098
[30] SHENG W, CHEN C, DONG M, WANG G, ZHOU J et al. Calreticulin promotes EGF-induced EMT in pancreatic cancer cells via Integrin/EGFR-ERK/MAPK signaling pathway. Cell Death Dis 2017; 8: e3147. https://doi.org/10.1038/cddis.2017.547

[31] LI C, GAO S, LI X, LI C, MA L. Procaine Inhibits Proliferation and Migration of Colon Cancer Cells Through Inactivation of the ERK/MAPK/FAK Pathways By Regulation of RhoA. Oncol Res 2018; 26: 209-217. https://doi.org/10.3727 /096504017X14944585873622

[32] SHENG W, CHEN C, DONG M, WANG G, ZHOU J et al. Calreticulin promotes EGF-induced EMT in pancreatic cancer cells via Integrin/EGFR-ERK/MAPK signaling pathway. Cell Death Dis 2017; 8: e3147. https://doi.org/10.1038/cddis.2017.547

[33] HUA L, QIAN M, XU CW, HUANG JH, ZHOU YF et al. OTX1 Contributes to Hepatocellular Carcinoma Progression by Regulation of ERK/MAPK Pathway. J Korean Med Sci 2016; 31: 1215-1223. https://doi.org/10.3346/ jkms.2016.31.8.1215

[34] YANG YC, MA YL, LIU WT, LEE EHY. Lamin-B1 Impairs Spatial Learning through Inhibition of ERK/MAPK and SGK1 Signaling. Neuropsychopharmacology 2011; 36: 2571-2586. https://doi.org/10.1038/npp.2011.148

[35] TAPIA O, RIQUELME I, LEAL P, SANDOVAL A, AEDO S et al. The PI3K/AKT/mTOR pathway is activated in gastric cancer with potential prognostic and predictive significance. Virchows Arch 2014; 465: 25-33. https://doi.org/10.1007/ s00428-014-1588-4

[36] KONG L, SCHÄFER G, BU H, ZHANG Y, ZHANG Y et al. Lamin A/C protein is overexpressed in tissue-invading prostate cancer and promotes prostate cancer cell growth, migration and invasion through the PI3K/AKT/PTEN pathway. Carcinogenesis 2012; 33: 751-759. https://doi.org/10.1093/ carcin/bgs022 